\title{
THE INTRINSIC CONFORMAL STRUCTURE AND GAUSS MAP OF A LIGHT-LIKE HYPERSURFACE IN MINKOWSKI SPACE
}

\author{
MAREK KOSSOWSKI
}

\begin{abstract}
We begin by pointing out two subtleties in the global properties of hypersurfaces in Minkowski space which inherit a uniformly degenerate metric (i.e., the existence of global space-like sections and the notion of an icon; see Appendices 1 and 2). We then construct a Gauss map for such hypersurfaces and an intrinsic invariant. This leads us to results concerning light-like hypersurfaces which parallel known results concerning surfaces in Euclidean space.
\end{abstract}

\section{INTRODUCTION}

A hypersurface in Minkowski space is light-like if it is everywhere tangent to an ambient light cone. (The induced metric is everywhere degenerate and the hypersurface is necessarily noncompact.) In this paper we construct an intrinsic conformal structure on such a hypersurface, show that it is a complete invariant for local isometry, and then link it with the degree of an associated Gauss map. As a result we have a degree formula, a characterization of the simplest lightlike hypersurface and several insights into the more subtle properties of such hypersurfaces.

There is a simple interrelationship between light-like hypersurfaces, spacelike 2-surfaces and compact hypersurfaces in Minkowski space. In this paper (and in [K3]) the relationship between the first two types of submanifolds is emphasized. However the degree formula established here will play a role in [K4] where we prove a hybrid degree formula for compact hypersurfaces in Minkowski space. One of the links in this interrelationship is the intrinsic conformal structure. This tensor appears as a natural consequence of a "Fundamental lemma of pseudo-Riemannian geometry" and is unique to degenerate pseudo-Riemannian metrics. Thus another motive for this paper is to provide a geometric interpretation for this tensor in the context of light-like hypersurfaces (compare [K1 and $\mathrm{K} 2]$ for interpretations in other settings). The author would like to thank R. Bryant, R. Harvey, R. Penrose and R. O. Wells for inspiration.

Received by the editors May 26, 1987 and, in revised form, May 3, 1988.

1980 Mathematics Subject Classification (1985 Revision). Primary 53C20, 53C22, 53C42, 57R45.

The author's research was partially supported by National Science Foundation Grants DMS 8382009 and DMS 8405207. 
An outline of this paper is as follows: We begin with an informal discussion of light-like hypersurfaces which points out several subtleties. This leads to a more formal definition of such a hypersurface and its associated Gauss map. We then introduce the intrinsic conformal structure and extract an essential invariant which appears in the degree formula. This is followed by the proof of the formula and several related results. The paper closes with several Appendicies which highlight examples and basic observations concerning light-like hypersurfaces.

Throughout this paper $\mathbf{M}^{4}$ will denote Minkowski space, the real fourdimensional vector space equipped with bilinear form $\langle$,$\rangle of type (3,1)$, (i.e. the normal form has three-plus signs and one-minus sign). We will assume that $\mathbf{M}^{4}$ is oriented and time oriented (i.e. a 4-volume form $d V$, and future time-like vector field Fut have been chosen). Thus when we speak of congruent hypersurfaces it will be relative to the orientation and time orientation preserving isometries of $\mathbf{M}^{4}$ (i.e., the group $\operatorname{SO}^{F}(3,1)$ ). It will be apparent to the reader that the arguments in this paper generalize in a straightforward manner to Minkowski space of any even dimension.

\section{Preliminary discussion}

We begin by provisionally defining a light-like hypersurface in $\mathbf{M}^{4}$ to be a smooth immersed orientable hypersurface in $\mathbf{M}^{4}$ such that the induced metric is everywhere degenerate (i.e., a type $(2,0)$ metric on a 3 -fold). Hence the hypersurface is fibered by curves which are tangent to the degenerate direction of the induced metric. A computation shows that these curves correspond to null (i.e., light-like) geodesic in $\mathbf{M}^{4}$. Thus light-like hypersurfaces are ruled by null geodesics and can be naturally enlarged by extending these ruling lines to complete geodesics. The price of this extension is that (in general) the hypersurface will no longer be immersed. Accepting this and keeping ruled surfaces in mind we would then hope for an orientable compact 2-surface within the hypersurface which is everywhere transverse to the ruling null geodesics. Such a 2-surface would then be immersed and space-like (i.e. it would inherit a positive definite metric). Our first observation is that there are extended light-like hypersurfaces in $\mathbf{M}^{4}$ which fiber over a 2-manifold but do not admit such a space-like transverse section (the construction of such a hypersurface is the content of Appendix 1). On the other hand, if we start with a smooth immersed orientable space-like 2-surface $k: M \rightarrow \mathbf{M}^{4}$, then the normal bundle is a trivial rank-2 bundle with type $(1,1)$ metric. And the two null subbundles (of rank1) may be viewed as a light-like hypersurface in $\mathbf{M}^{4}$. Thus we have a simple interrelationship: every space-like 2-surface may be viewed as the intersection of two associated light-like hypersurfaces and light-like hypersurface (which admits a space-like section) maybe viewed as a subbundle of this section's normal bundle. 
We now provide a more formal definition of light-like hypersurface. Motivated by the discussion above, let $M$ be a smooth compact oriented 2-manifold.

Definition 1. A light-like hypersurface $N$ with base $M$ is a smooth map $i: M \times$ $\mathbf{R}=N \rightarrow \mathbf{M}^{4}$ (nonimmersive on the set $S(i) \subset N$, ) such that

(a) for all $p \in N-S(i)$ the type of the induced metric $i^{*}\langle,\rangle_{p}=\overline{\mathbf{I}}_{p}$ is $(2$, $0)$

(b) the fibers of $\pi: N \rightarrow M$ are affinely parametrized complete null geodesics in $\mathbf{M}^{4}$ which are $\overline{\mathrm{I}}_{p}$-orthogonal to all of $T_{p} N$,

(c) there exists a smooth section $r$ of $\pi: N \rightarrow M$ which does not intersect $S(i)$.

(We remark that in Appendix 1 we construct an example of such a map which satisfies (a) and (b) but not (c).) A section $r$ of $\pi: N \rightarrow M$ which satisfies condition (c) will be called a space-like section of $N$ (since a section $r$ yields a space-like immersion $k: M \rightarrow \mathbf{M}^{4}$, where $k=i \circ r$ if and only if $r(M) \cap S(i)$ is empty). To discuss orientation suppose that we have chosen $t: N \rightarrow \mathbf{R}$ as a trivialization of $\pi$ such that $i_{*} \partial t \neq 0$ is future pointing (i.e. $\left\langle i_{*} \partial t\right.$, Fut $\rangle<0$ ). If $M$ is oriented by a 2 -form $d A$ then $\pi^{*} d A \wedge d t$ orients $N$. It is of note that $i_{*}$ does not respect orientation, that is to say,

$$
i^{*} d V(-,-,-, \text { Fut }) \text { and } \pi^{*} d A \wedge d t
$$

need not induce the same orientation on $N$. In fact the "sign" of $i^{*} d V(-,-$, Fut $)$ will differ on the various components of $N-S(i)$. We may visualize matters as in Figure 1.

We will say that a light-like hypersurface is an icon if there exists a spacelike affine hyperplane $\mathbf{E}^{3} \subset \mathbf{M}^{4}$ which intersects the extended hypersurface in an immersed copy of $M$ (i.e. the hyperplane misses the nonimmersed points $S(i))$. We refer to such an intersection as a hyperplane section of the light-like hypersurface. Every immersed point of a light-like hypersurface admits local
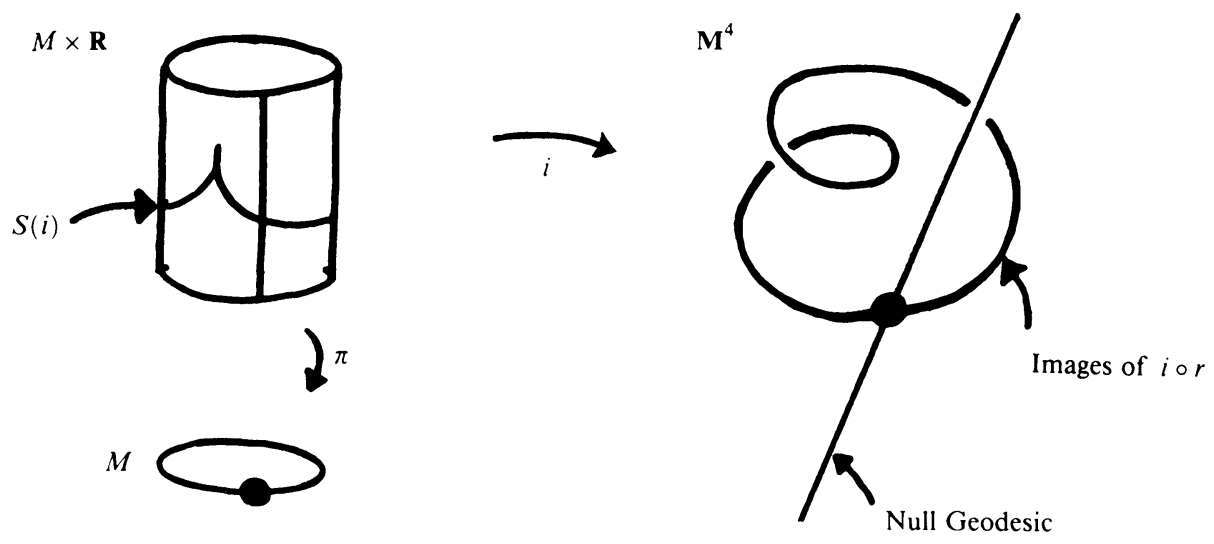

Figure 1 
hyperplane sections; however in Appendix 2 we construct a light-like hypersurface which is not an icon. This is a key point, since many of the results of this paper would otherwise be immediate consequences of the Euclidean geometry of surfaces in $\mathbf{E}^{3}$.

We now define a Gauss map, $g: M \rightarrow S^{2}$, associated to a light-like hypersurface $(N, i)$ with base $M$. Let $r$ be any section of $\pi: N \rightarrow M$. Then for $x \in M, i\left\{\pi^{-1}(x)\right\}$ is a null line containing $i \circ r(x) \in \mathbf{M}^{4}$. Thus by translating this point to the origin in $\mathbf{M}^{4}$ we get an oriented null line $n_{x}$ lying in the light cone $\mathbf{L C}=\left\{v \in \mathbf{M}^{4} \mid\langle v, v\rangle=0\right\}$. Since the light cone is a light-like hypersurface with base $S^{2}$ and projection $\bar{\pi}: \mathrm{LC} \rightarrow S^{2}$, we define $g(x)$ to be the point $\bar{\pi}\left(n_{x}\right) \in S^{2}$. To see that $g$ does not depend on the choice of section one need only observe that if $r_{1}$ and $r_{2}$ are any two sections, then $i \circ r_{1}(x)$ and $i \circ r_{2}(x)$ lie on the same null line.

To make matters more concrete choose a space-like section $r$ and an orthogonal splitting $\mathbf{M}^{4}=\mathbf{E}^{3}+\mathbf{E}^{-}$, with projection $t: \mathbf{E}^{3}+\mathbf{E}^{-} \rightarrow \mathbf{E}^{-}$( $t$ is assumed to increase in the future direction). Now, let $R$ be the vector field on $N$ tangent to the fibers of $\pi$ such that $t_{*}(R)$ is everywhere of unit length. Then the Gauss map $g$ is represented by $R \circ r$ where the target $S^{2}$ is represented by the intersection $\operatorname{LC} \cap\left\{t^{-1}(1)\right\}$.

Suppose now that we start with an oriented space-like immersion $k: M \rightarrow$ $\mathbf{M}^{4}$. Since the normal bundle $M$ is a trivial rank two bundle with type $(1,1)$ metric there exist two unique (modulo $\operatorname{SO}^{F}(1,1)$ ) null sections $R^{F}, R^{P}$ of $M^{\perp}$ which satisfy

$$
\begin{aligned}
& \left\langle R^{F}, R^{P}\right\rangle=1, \quad\left\langle R^{F}, \text { Fut }\right\rangle<0, \\
& \left\langle R^{i}, R^{i}\right\rangle=0, \quad i=F, P, \\
& d V\left(-,-, R^{P}, R^{F}\right)=d A(-,-), \quad(d A \text { is the area form of }(M, k)) .
\end{aligned}
$$

The two light-like hypersurfaces associated with $(M, k)$ are given by

$$
i^{F}(x, r)=k(x)+r R^{F}(x), \quad i^{P}(x, r)=k(x)-r R^{P}(x),
$$

and will be denoted $\mathrm{LL}^{i}(M), i=F, P$. We now have $g^{i}: M \rightarrow S_{i}^{2}, i=F, P$, two $S^{2}$-valued Gauss maps which are uniquely determined by the orientation of $M$. For a concrete representation of these maps choose an orthogonal splitting $\mathbf{M}^{4}=\mathbf{E}^{3}+\mathbf{E}^{-}$with projections $t: \mathbf{E}^{3}+\mathbf{E}^{-} \rightarrow \mathbf{E}$ and $\pi_{\mathbf{E}}: \mathbf{E}^{3}+\mathbf{E}^{-} \rightarrow \mathbf{E}^{3}$. Now let $U^{i}$ be the positive multiple of $R^{i}, i=F, P$, such that $t_{*} U^{i}$ is of unit length. Then the Gauss map $g^{i}$ is represented by $U^{i}: M \rightarrow S_{i}^{2}, i=F, P$, where

$$
S_{F}^{2}=\operatorname{LC} \cap\{t=1\}, \quad S_{P}^{2}=\operatorname{LC} \cap\{t=-1\},
$$



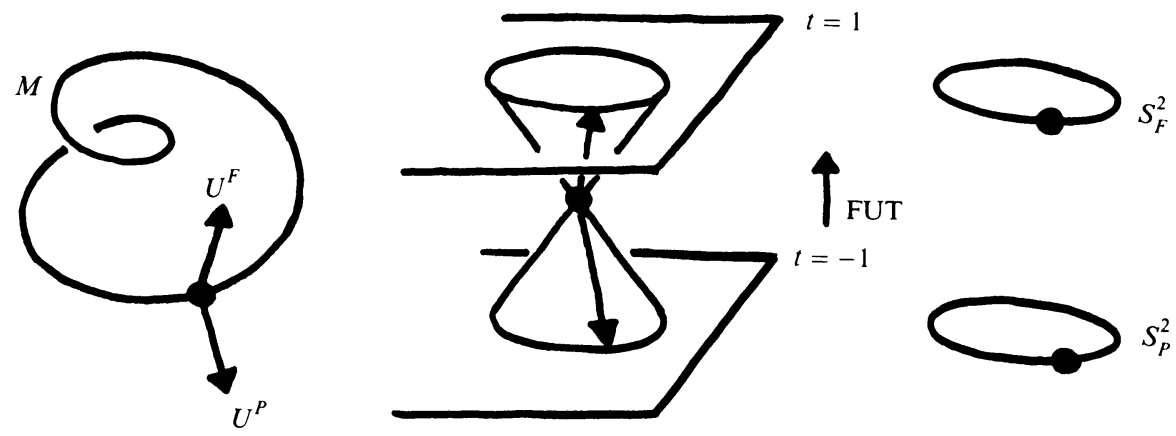

Figure 2

We will assume that these $S_{i}^{2}, i=F, P$, are oriented so that the null sections of $S_{i}^{2}$, given by (E2), project via $\pi_{E}: \mathbf{M}^{4} \rightarrow \mathbf{E}^{3}$ to the outward normal of the unit sphere in $\mathbf{E}^{3}$. We may visualize matters as in Figure 2.

\section{THE INTRINSIC CONFORMAL STRUCTURE}

Here we define the intrinsic conformal structure of a light-like hypersurface and present two interpretations. We define this invariant by way of a dual version of the fundamental lemma of pseudo-Riemannian geometry which is valid even when the pseudo-Riemannian metric is degenerate. The intrinsic conformal structure then appears naturally as an invariant of the induced (degenerate) metric on a light-like hypersurface. It should be emphasized that this invariant is determined entirely by the induced metric on the hypersurface. (Appendix 4 indicates that generically it is a complete invariant). An extrinsic interpretation for this invariant is gotten by way of local hyperplane sections of the light-like hypersurface. This establishes a strong link between light-light hypersurfaces and surfaces in Euclidean three space. The remainder of the paper develops this link.

Keeping $i^{*}\langle\rangle=,\mathrm{I}$ in mind let $(N,\langle\rangle$,$) be a smooth manifold with \langle$, any smooth symmetric $(0,2)$ tensor. The symbols $\mathfrak{X}(N), \Omega(N)$ and $C^{\infty}(N)$ will denote the smooth vector fields, 1-forms and real-valued functions on $N$, respectively.

Definition 2. Given $(N,\langle\rangle$,$) , a C^{\infty}$ dual connection on $N$ is a map

$$
\begin{aligned}
\square: \mathfrak{X}(N) \times \mathfrak{X}(N) & \rightarrow \Omega(N) \\
X, Y & \rightarrow \square_{X} Y
\end{aligned}
$$

which satisfies

(a) $\square$ is $\mathbf{R}$ bilinear;

(b) for all $f \in C^{\infty}(N)$ and $X, Y \in \mathfrak{X}(N)$

(1) $\square_{f X} Y=f \square_{X} Y$,

(2) $\square_{X} f Y=X(f)\langle Y,-\rangle+f \square_{X} Y$. 
The torsion of $\square$ is the $(0,3)$ tensor

$$
\operatorname{Tor}(X, Y, Z)=\square_{X} Y(Z)-\square_{Y} X(Z)-\langle[X, Y], Z\rangle ;
$$

here $\square_{X} Y(Z)$ denotes the pairing of the 1 -form $\square_{X} Y$ with the vector field $Z$. is compatible with \langle\rangle if

$$
X\langle Y, Z\rangle=\square_{X} Y(Z)+\square_{X} Z(Y)
$$

Lemma 3. Given $(N,\langle\rangle)$ there exists a unique torsion free compatible dual connection.

Proof. As in the classical case we use the relations of compatibility and Tor $=0$ to write

$$
\begin{aligned}
2 \square_{X} Y(Z)= & X\langle Y, Z\rangle+Y\langle Z, X\rangle-Z\langle X, Y\rangle \\
& +\langle[X, Y], Z\rangle-\langle[X, Z], Y\rangle-\langle[Y, Z], X\rangle .
\end{aligned}
$$

One then shows that this object satisfies (a) and (b).

Observe that if $\langle$,$\rangle is nondegenerate then \square_{X} Y(Z)=\left\langle\nabla_{X} Y, Z\right\rangle$, where $\nabla$ is the classical Levi-Civita connection. If $\langle,\rangle_{p}$ is degenerate, let $\operatorname{Rad}\left(\langle,\rangle_{p}\right)$ denote the subspace of $T_{p} N$ which is $\langle,\rangle_{p}$-orthogonal to all of $T_{p} N$. Now let $R \in \operatorname{Rad}\left(\langle,\rangle_{p}\right)$ and $X_{p}, Y_{p} \in T_{p} N$ and set

$$
\overline{\mathrm{II}}_{p}(X, Y, R)=\square_{X_{p}} Y(R) \text {. }
$$

Proposition 4. (a) $\overline{\mathrm{II}}_{p}$ is a tensor on $T_{p} N \times T_{p} N \times \operatorname{Rad}\left(\langle,\rangle_{p}\right)$.

(b) $\overline{\mathrm{II}}_{p}(X, Y, R)=\overline{\mathrm{II}}_{p}(Y, X, R)$.

Proof. (a) Observe; $\square_{X_{p}} f Y(R)=X(f)\langle Y, R\rangle_{P}+f \square_{X_{p}} Y(R)$ and $\langle Y, R\rangle_{p}=0$.

(b) Observe; $0=\langle R,[X, Y]\rangle_{p}=\square_{X_{p}} Y(R)-\square_{Y_{p}} X(R)$.

We now carry out this construction for the pair $(N-S(i), \overline{\mathrm{I}})$. Notice that $\operatorname{Rad}\left(\overline{\mathbf{I}}_{p}\right)=\operatorname{Ker} \pi_{* p}$ is one dimensional and there is no natural choice for $R \in$ $\operatorname{Rad}\left(\overline{\mathrm{I}}_{p}\right)$. Thus we view $\overline{\mathrm{II}}_{p}(-,-, R)$ is a conformal structure, the intrinsic conformal structure of a light-like hypersurface. The simplest extrinsic interpretation for $\overline{\mathrm{II}}$ is given by noting that if $v, u \in T_{p} N$ are transverse to $\operatorname{Rad}\left(\overline{\mathrm{I}}_{p}\right)$, then

$$
\overline{\mathrm{II}}_{p}(u, v, R)={ }^{-}\left\langle\nabla_{u} R, v\right\rangle_{p} \quad(\nabla \text { is the ambient connection }) .
$$

So if $R$ represents the Gauss map $g$ of $(N, i)$ we see that $\overline{\mathrm{II}}_{p}$ can be used to reconstruct the differential of $g$. A simple interpretation for $\overline{\text { II }}$ in the case of a space-like two-surface $(M, k)$ is given by choosing $R^{F}, R^{P}$ as in (E2). Then the vector valued second fundamental form II $^{\perp}$ of $(M, k)$ decomposes as

$$
\mathrm{II}^{\perp}(-,-)=\overline{\mathrm{II}}^{F}\left(-,-, R^{F}\right) R^{P}+\overline{\mathrm{II}}^{P}\left(-,-, R^{P}\right) R^{F},
$$

where $\overline{\mathrm{II}}^{i}$ is the intrinsic conformal structure for $\mathrm{LL}^{i}(M), i=F, P$. Appendix 4 contains another interpretation for $\overline{\mathrm{II}}$. 
Now, returning to the light-like hypersurface setting and examining (E4), it is immediate that for all $R \in \operatorname{Rad}\left(\overline{\mathrm{I}}_{p}\right)$ the covector $\overline{\mathrm{II}}_{p}(-, R, R)$ is zero. Hence both $\overline{\mathrm{I}}_{p}$ and $\overline{\mathrm{II}}_{p}$ are well defined on the rank two bundle $T N / \operatorname{Rad}(\overline{\mathrm{I}})$ over $N-S(i)$. We define the index of $(N-S(i), \overline{\mathrm{I}})$ at $p$ to be the index of the bilinear form $\overline{\mathrm{II}}_{p}$ on the vector space $T_{p} N / \operatorname{Rad}\left(\overline{\mathrm{I}}_{p}\right)$, and $\operatorname{sign}(p)=(-1)^{\text {index }}$. It will be convenient to extract an invariant function from $\overline{\mathrm{II}}$ which encodes $\operatorname{sign}(p)$. Unfortunately it will be necessary to impose a regularity condition which prohibits $(N, i)$ from being "too flat" (compare Proposition 6(a)). If we fix $R \in \operatorname{Rad}\left(\mathrm{I}_{p}\right)$ we may speak of the length of $\overline{\mathrm{II}}_{p}(-,-, R)$ relative to $\overline{\mathrm{I}}_{p}$, i.e. $\|\overline{\mathrm{I}}(-,-, R)\|_{\overline{\mathrm{I}}}$.

We impose the following regularity condition: there exists a section $R$ so that $\|\overline{\mathrm{I}}(-,-, R)\|_{\overline{\mathrm{I}}}$ does not vanish on $N-S(i)$. Hence there exists a unique (up to multiplication by \pm ) section $\bar{R}$ of $\operatorname{Rad}(\overline{\mathrm{I}})$ such that

$$
\|\overline{\mathrm{II}}(-,-, \bar{R})\|_{\overline{\mathrm{I}}}=1 \text {. }
$$

It follows that $T N / \operatorname{Rad}(\overline{\mathrm{I}})$ carries $\overline{\mathrm{I}}$ and $\overline{\mathrm{II}}(-,-, \bar{R})$, and that we may define two functions on $N-S(i)$.

Definition 5. For $p \in N-S(i)$ and $X_{1}, X_{2} \in T_{p} N$ such that $X_{1}, X_{2}, \pm \bar{R}(p)$ frame $T_{p} N$, set

$$
\bar{K}(p)=\frac{\operatorname{DET} \overline{\mathrm{II}}_{p}\left(X_{i}, X_{j}, \bar{R}\right)}{\operatorname{DET} \overline{\mathrm{I}}_{p}\left(X_{i}, X_{j}\right)}, \quad \bar{H}_{(p)}=\frac{\operatorname{TRACE} \overline{\mathrm{II}}_{p}\left(X_{i}, X_{j}, \bar{R}\right)}{2 \operatorname{DET} \overline{\mathrm{I}}_{p}\left(X_{i} X_{j}\right)} .
$$

Notice that $\bar{K}(p)$ is independent of the choice of $\pm \bar{R}$ and the $X_{i}$, and $\operatorname{sign}(p)$ agrees with the sign of $\bar{K}(p)$, whereas $\bar{H}(p)$ changes sign with the choice of $\pm \bar{R}$.

Let us interpret $\overline{\mathrm{II}}$ and $\bar{K}(p)$ in terms of a local space-like hyperplane section. If $\mathbf{E}^{3} \subset \mathbf{M}^{4}$ is any space-like hyperplane containing $p \in N-S(i)$ then near $p$ the intersection is an imbedded hypersurface in $\mathbf{E}^{3}$. We denote the first and second fundamental forms for this Euclidean hypersurface by I and II, the Gaussian curvatures by $K$. We remind the reader that these objects depend upon the choice of hyperplane through $p$.

Proposition 6. For any space-like hyperplane section containing $p \in N-S(i)$,

$$
\begin{aligned}
& \overline{\mathrm{II}}_{p}=\frac{ \pm 1}{\|\mathrm{II}\|} \mathrm{II}_{p}, \\
& \bar{K}(p)=\frac{K}{\|\mathrm{II}\|^{2}}(p) .
\end{aligned}
$$

Proof. Let $j: U \subset \mathbf{R}^{2} \rightarrow \mathbf{E}^{3}, j(0)=p$ be a local parametrization of the hyperplane section, and let $\omega_{1}, \omega_{2} \in T_{0}^{*} U$ be an orthonormal coframe such that $\mathrm{II}_{0}=\kappa_{1} \omega_{1} \circ \omega_{1}+\kappa_{2} \omega_{2} \circ \omega_{2}$. Near $p$, we may locally parametrize the light-like hypersurface as $i: U \times \mathbf{R} \rightarrow \mathbf{E}^{3}+\mathbf{E}^{-}, i(x, r)=j(x)+r \cdot(n(x), 1)$, 
where $n$ is a choice of normal in the hyperplane section. Setting $\alpha=\left(1+r \kappa_{1}\right)$ and $\beta=\left(1+r \kappa_{2}\right)$, we compute:

$$
\begin{gathered}
\overline{\mathrm{I}}_{(0, r)}=\alpha^{2} \omega_{1} \circ \omega_{1}+\beta^{2} \omega_{2} \circ \omega_{2}, \\
\overline{\mathrm{II}}_{(0, r)}(-,-, \partial r)=\kappa_{1} \alpha \omega_{1} \circ \omega_{1}+\kappa_{2} \beta \omega_{2} \circ \omega_{2}, \\
\|\overline{\mathrm{I}}(-,-, \partial / \partial r)\|_{\overline{\mathrm{I}}}=\left(\frac{\left(\kappa_{1} \beta\right)^{2}+\left(\kappa_{2} \alpha\right)^{2}}{\alpha^{2} \beta^{2}}\right)^{1 / 2} .
\end{gathered}
$$

Thus

$$
\begin{aligned}
& \bar{R}_{(0, r)}=\left(\frac{\alpha^{2} \beta^{2}}{\left(\kappa_{1} \beta\right)^{2}+\left(\kappa_{2} \alpha\right)^{2}}\right)^{1 / 2} \partial r, \\
& \bar{K}_{(0, r)}=\frac{\kappa_{1} \kappa_{2} \alpha \beta}{\left(\kappa_{1} \beta\right)^{2}+\left(\kappa_{2} \alpha\right)^{2}},
\end{aligned}
$$

and evaluating at $(0,0)$ we obtain $(a)$ and $(b)$.

Also note that (E9) shows us that a section $r$ is space-like iff $r(M)$ does not intersect the singular locus $S(i)$.

Now examining (E13) and noticing that $\alpha \beta$ vanishes exactly where the $\pi: N \rightarrow M$ fibers intersect $S(i)$ one observes the following.

Proposition 7. Given $(N-S(i), \overline{\mathrm{I}})$ satisfying the regularity conditions (E7):

(a) $\bar{K}$ extends smoothly to all of $N$.

(b) $\bar{K} \leq 1 / 2$ on $N$ and $\bar{K}(p)=0$ if $p \in S(i)$.

(c) If $\bar{K}(p)>0($ resp. $<0)$, then $\bar{K}$ is positive (resp. negative) on the intersection of the fiber through $p$ with the connected component of $N-S(i)$ which contains $p$.

(d) If $\bar{K}(p)=1 / 2$ then $\bar{K}$ is constant on the entire fiber through $p$.

\section{The Degree Formula AND Related Results}

Theorem 8. (a) Given a light-like hypersurface $\pi: N \rightarrow M$ with $M$ oriented, then the Gauss map has degree $\pm \chi(M) / 2$.

(b) For any space-like immersion $k: M \rightarrow \mathbf{M}^{4}$ the Gauss maps $g^{i}, i=F, P$, have degree $\chi(M) / 2$.

Remarks. The ambiguity in the sign of part (a) is a consequence of (E1) (i.e. there is no consistant way of linking the Gauss map of $(N, i)$ with the orientation of $M$ ). Recall that for part (b) the orientation of $M$ determines the Gauss maps via (E2). Also recall that the target spheres $S_{i}^{2}, i=F, P$, carry preferred orientations.

Proof. (a) With a choice of space-like section, part (a) follows from part (b).

(b) Choose a splitting $\mathbf{M}^{4}=\mathbf{E}^{3}+\mathbf{E}^{-}$. We view the Gauss maps as taking values in the spheres $S_{i}^{2}, i=F, P$ (see (E3)). Consider the homotopy $k_{s}=$ 
$H_{s} \circ k$, where $H_{s}: \mathbf{M}^{4} \times[0,1] \rightarrow \mathbf{M}^{4}$, with $H_{s}(x, t)=(x, s t), x \in \mathbf{E}^{3}, t \in \mathbf{E}^{-}$ and $s \in[0,1]$. We see that $k_{s}$ is a space-like immersion, $k_{1}=k$ and $k_{0}$ is an immersion of $M$ into $\mathbf{E}^{3}$. Further the $\left(H_{s}\right)_{*} \circ U^{i}, i=F, P$, take values in spheres which can be identified (by way of $\pi_{\mathrm{E}}$ ) with the outward oriented unit sphere in $\mathbf{E}^{3}$. Thus $\left(H_{0}\right)_{*} U^{i}, i=F, P$, is identified with the $\mathbf{E}^{3}$ Gauss map of $k_{0}: M \rightarrow \mathbf{E}^{3}$, and our two Gauss maps are homotopic to the $\mathbf{E}^{3}$ Gauss map of $k_{0}: M \rightarrow \mathbf{E}^{3}$. By the $\mathbf{E}^{3}$ Gauss-Bonnet theorem we are finished.

Corollary 9. If the genus of $(M, k)$ is nonzero then the Gauss maps $g^{i}: M \rightarrow$ $S_{i}^{2}, i=F, P$, are surjective.

In Appendix 3 we construct an example of an embedded space like a 2-torus which has both Gauss maps $g^{i}, i=F, P$, nonsurjective.

Given $(N, i)$, if $n \in S^{2}$ is a regular value of the Gauss map $g: M \rightarrow S^{2}$, then the set of $\pi: N \rightarrow M$ fibers over $\left\{g^{-1}(n)\right\}$ will denoted $\mathrm{FO}(n)$. Since $M$ is compact, $\left\{g^{-1}(n)\right\}$ is a finite set. Given a regular value $n$ and a section $r$ of $\pi: N \rightarrow M$ we get a (possibly empty) intersection $r(M) \cap \mathrm{FO}(n)$ of the image of the section $r(M)$ and the fibers over $\left\{g^{-1}(n)\right\}$. We also have the sign of $(n, i)$ at each point of $r(M) \cap \mathrm{FO}(n)$.

Corollary 10. For all space like sections $r$ of $(N, i)$ and regular values $n$ of $g$,

$$
\sum_{p \in r(M) \cap \mathrm{FO}(n)} \operatorname{sign}(p)= \pm \chi(M) / 2 .
$$

Proof. Since $(M, k)$, where $k=i \circ r$, is space-like, the Gauss map of $(N, i)$ agrees with one of the Gauss maps $g^{i}$ of $(M, i)$, or agrees with (anti) $\circ g^{i}$ where anti: $S_{i}^{2} \rightarrow S_{i}^{2}$ is the antipodal map. Thus by part (b) of Theorem 9 we are done.

We now draw several conclusions.

Theorem 11. There exists no light-like hypersurface with $\bar{K}<0$ on a space-like section of $\pi: N \rightarrow M$.

Proof. If there was such a section, (E9) and (E10) would give a global framing of $M$ by the principal directions of $\overline{\mathrm{II}}$. Hence $M$ must be a 2-torus. Now we choose a nonvacuous regular value for the Gauss map of $(N, i)$ and recall that the signs of $\bar{K}(p)$ and $\operatorname{sign}(p)$ agree. Thus Corollary 10 would imply $\chi(M) \neq 0$ and we have a contradiction.

Corollary 12. Given a space-like surface $(M, k)$, then both $\bar{K}^{i}, i=F, P$, take a nonnegative value on $M$.

Proof. Apply Theorem 11 to both of the $\operatorname{LL}^{i}(M), i=F, P$.

Remark. Upon reflection the reader will observe that there is a simple extrinsic proof that one of the $\bar{K}_{i}$ in Corollary 12 must take a nonnegative value on $M$. The issue of the existence of positive values will be further discussed in [K3]. 
Theorem 13. If $\bar{K}>0$ on a space-like section of $\pi: N \rightarrow M$, then $(N, i)$ is congruent to an icon over a convex sphere which lies in a hyperplane $\mathbf{E}^{3} \subset \mathbf{M}^{4}$. Proof. We note that the compactness of $M, \bar{K}>0$ and equation (E9) implies that $S(i)$ is $\mathbf{E}^{4}$-bounded in $\mathbf{M}^{4}$. Thus choosing a hyperplane beyond $S(i)$ we have a hyperplane section. Hence $(N, i)$ is an icon. Now if this hyperplane section was not strictly convex then there would be a point of zero Gauss curvature on this hyperplane section. But Proposition 7 would then imply that $\bar{K}$ would vanish on the entire fiber of $\pi: N \rightarrow M$ through this point. This contradicts $\bar{K}>0$ on a section.

Corollary 14. If $(M, k)$ has both $\bar{K}^{i}, i=F, P$, strictly positive, then $(M, k)$ is congruent to the intersections of two icons on convex spheres.

Proof. Apply Theorem 13 to both the $\operatorname{LL}^{i}(M, k), i=F, P$.

Theorem 15. Any one of the following two conditions implies that $(N, i)$ is congruent to a light cone.

(a) $\bar{K}=1 / 2$ on a space-like section,

(b) $\bar{K}>0$ on $N-S(i)$.

Proof. By Theorem 13 we know $(N, i)$ is an icon. Now examining a hyperplane section and Propositions 6 and 7 we see that any hyperplane section is totally umbilic and hence a round $S^{2} \subset \mathbf{E}^{3} \subset \mathbf{M}^{4}$.

Corollary 16. A space-like surface $(M, k)$ with both $\bar{K}^{i}=1 / 2, i=F, P$, is congruent to a round 2-sphere contained in a space-like hyperplane of $\mathbf{M}^{4}$.

Proof. Apply Theorem 15 to both the $\operatorname{LL}^{i}(M), i=F, P$, to conclude that $(M, k)$ is the intersection of two light cones. Since the intersection must be space-like and compact we are finished.

Corollary 17. A space-like surface $(M, k)$ with one $\bar{K}^{i}=1 / 2$ is embedded.

Proof. By Theorem $15(M, k)$ is a space-like section of a light cone and hence is embedded.

Remarks. Upon examining space-like sections of an icon over an ellipsoid one readily sees that the positivity (or pinching $0<c \leq \bar{K}^{i} \leq 1 / 2$ ) of one of the $\bar{K}^{i}$ on a section does not imply that the section is embedded. However in Appendix 1 of [K3] we show that if both of the $\bar{K}^{i}, i=F, P$, are positive on $(M, k)$, then the space-like surface is embedded.

The results described above suggest further comparisons between the Gauss maps of surfaces in $\mathbf{E}^{3}$ and in $\mathbf{M}^{4}$. This will be the subject of [K3]. We close this paper with several observations.

\section{APPENDIX 1}

Our purpose here is to construct an example of light-like hypersurface in $\mathbf{M}^{4}$ which satisfies conditions (a) and (b) but not (c) of Definition 1. The example presented here will have base $S^{2}$ and will admit a section which is immersed 
but not space-like. The obstruction to a space-like section will be supplied by our degree formula for the Gauss map. We begin the construction. Consider the graph of $t=x^{2} / 2+y^{2} / 2+z^{3} / 3$ in split Minkowski space $\mathbf{M}^{4}=\mathbf{E}^{3}+\mathbf{E}^{-}$. The induced metric on the graph is degenerate exactly over the 2 -sphere,

$$
1=(x)^{2}+(y)^{2}+\left(z^{2}\right)^{2} .
$$

Now the line orthogonal to this graph in $\mathbf{M}^{4}$ is spanned by $R=\left(x, y, z^{2}, 1\right)$ and is tangent to the graph over the 2-sphere (A1). Consider the light-like hypersurface

$$
\begin{aligned}
i: S^{2} \times \mathbf{R} & \rightarrow \mathbf{M}^{4}, \\
(p, r) & \rightarrow p+r R(p) .
\end{aligned}
$$

Conditions (a) and (b) of our definition follow from the fact that the metric induced on the graph is degenerate over the $S^{2}$. Also note that $R$ restricted to the $S^{2}$ represents the Gauss map of the light-like hypersurface. We claim that condition (c) of Definition 1 cannot hold. To see this recall our discussion of the Gauss map and its degree. We know that if there were such a space-like section the degree of the Gauss map would be \pm 1 . Further with a homotopy of the type used in the proof of Theorem 8 we see that the degree of the Gauss map agrees with the degree of the map of $S^{2}$ to $S^{2} \subset \mathbf{E}^{3}$ given by normalizing the vector field $X=\left(x, y, z^{2}\right)=x \partial x+y \partial y+z^{2} \partial z$. Now

$$
X_{s}=s X+(1-s) \partial z
$$

gives a homotopy of $X$ with the constant vector field. Hence the degree of the Gauss map is zero. So the light-like hypersurface (A2) does not admit a space-like section.

\section{APPENDIX 2}

Our purpose here is to construct an example of a light-like hypersurface in $\mathbf{M}^{4}$ which is not an icon. Using the intuition supplied by [K1], we will build an example of an immersed space-like 2-disk such that one of its light-like hypersurfaces is not an icon and thus convince the reader that local deformation can destroy an icon. In Appendix 3 of [K3] we will give a more indirect construction of such a hypersurface which illustrates how more subtle global properties can obstruct the existence of a hyperplane section.

The 2-disk will be a surface of revolution in $\mathbf{M}^{4}$. So we need only construct a 1-disk in $\mathbf{M}^{3}$ such that one of its light-like hypersurfaces is not an icon in $\mathbf{M}^{3}$. To this end choose a null 2-plane $\mathbf{N}^{2}$ in $\mathbf{M}^{3}$, which is a light-like hypersurface and hence ruled by null lines. Now any immersed curve $c$ in $\mathbf{N}^{2}$ which is transverse to this ruling is space like and has the chosen $\mathbf{N}^{2}$ as one of its lightlike hypersurfaces $\operatorname{LL}^{i}(c)$. By deforming this curve in $\mathbf{N}^{3}$ we will put a strong restriction on hyperplane sections of the other $\mathrm{LL}^{i}(c)$. The essential lesson of [K1] is that as the curve is deformed in $\mathbf{N}^{2}$ so that it becomes tangent to 

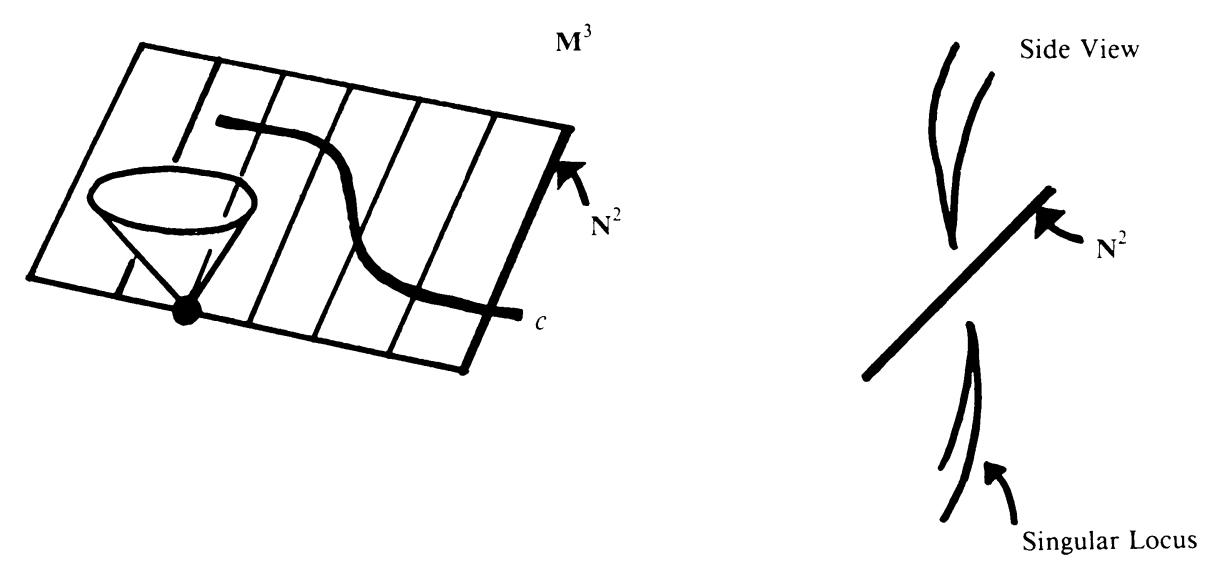

FigURE 3

a ruling line, the focal surface of the curve approaches the point of tangency. Now the singular loci of the $\operatorname{LL}^{i}(c)$ are just the intersections of the $\operatorname{LL}^{i}(c)$ with the focal surface of $c$. Hence a symmetric curve with "flat spots' has drastically constricted hyperplane sections (see Figure 3).

This curve's symmetry under reflection (about a null line in $\mathbf{N}^{2}$ ) forces the singular locus to lie on both sides of $\mathbf{N}^{2}$ and the flat spots force the singular locus to extend to both $\pm \infty$ (this obstructs "far away" hyperplane sections). Now if we distribute several copies of such a segment about $\mathbf{M}^{3}$ and join them with space-like segments the resulting space-like 1-disk will have one associated light-like hypersurface which does not admit a hyperplane section. Finally by closing this curve with a space-like segment and choosing an appropriate axis of rotation we may build an immersed $S^{2}$ or $T^{2}$ in $\mathbf{M}^{4}$ with at least one $\mathrm{LL}^{i}$ not an icon. It should be clear from the above that it is possible to construct compact $(M, k)$ of any genus, with exactly one $\operatorname{LL}^{i}(M)$ not an icon.

We close this appendix with an explicit example which illustrates the "essential lesson of [K1]" mentioned above. Consider a parametrized curve in $\mathbf{N}^{2} \subset \mathbf{M}^{3}$,

$$
\mathbf{R} \rightarrow\left(\mathbf{R}^{3}, d x^{2}+d y d z\right), \quad t \rightarrow(f(t), t, 0) .
$$

If $f^{\prime} \neq 0$, the curve is space-like. The "other" light-like hypersurface associated to this curve is parametrized by

$$
\mathbf{R}^{2} \rightarrow \mathbf{R}^{3}, \quad(t, r) \rightarrow(r+f, t,-r f),
$$

and its singular locus is given by the relation

$$
\left(f^{\prime}\right)^{2}+r f^{\prime \prime}=0 \text {. }
$$

Now the 1-parameter family of curves $c_{\lambda}$ given by $f_{\dot{\lambda}}(t)=t^{3} / 3+\lambda^{2} t$ is spacelike for all $t$ if $\lambda \neq 0$, and space-like for all $t \neq 0$ if $\lambda=0$. Now notice that $\left(-2 s^{3}, s, s\right)=(r, t, \lambda)$ is an immersed curve contained in the variety $\left(f_{\lambda}^{\prime}\right)^{2}+$ 
$r f_{\lambda}^{\prime \prime}=0$, which is transverse to $\{\lambda=0\}$. Hence as $\lambda \rightarrow 0$ the singular locus of the "other" light-like hypersurface $\operatorname{LL}^{i}\left(c_{\hat{\lambda}}\right)$ approaches the point $t=0$ on $c_{0}$.

\section{APPENDIX 3}

Our purpose here is to construct an imbedded space-like 2-torus in $\mathbf{M}^{4}$ such that one of its Gauss maps $g^{i}$ is not surjective (i.e. omits a set of full measure). This is in contrast to 2-surfaces in $\mathbf{E}^{3}$. The surface will be a 2-torus of revolution. A more detailed discussion of surfaces of revolution appears in Appendix 2 of [K3]. The profile curve will be a slight modification of the space-like imbedded circle $k: S^{1} \rightarrow \mathbf{M}^{3}$ depicted in Figure 4.

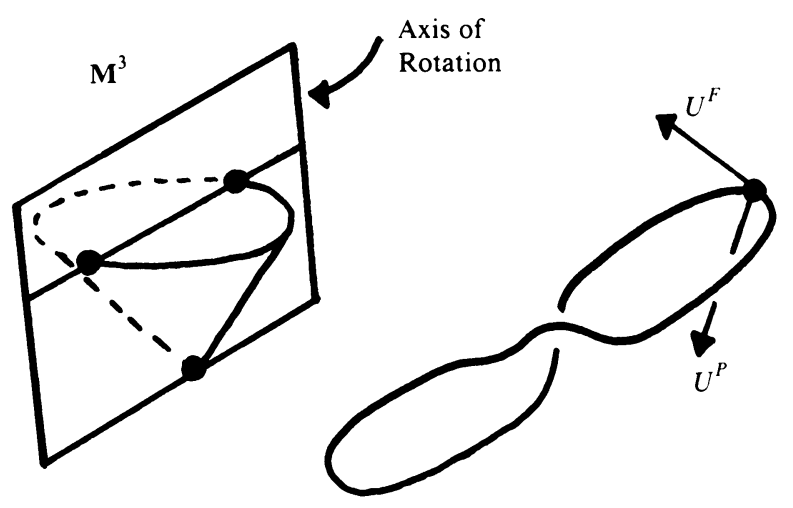

FigURE 4

The images of its two Gauss maps $g^{i}: S^{1} \rightarrow S_{i}^{1}, i=F, P$, are not surjective.

Now consider the composition of $k$ with the one-parameter family of isometries in $\mathbf{M}^{3}$ :

$$
\left(\begin{array}{ccc}
1 & 0 & 0 \\
0 & \cosh \theta & \sinh \theta \\
0 & \sinh \theta & \cosh \theta
\end{array}\right) .
$$

This yields space-like immersions $k_{\theta}: S^{1} \rightarrow \mathbf{M}^{3}$. One of the Gauss images of $k_{\theta}$ gets "smaller" as $\theta \rightarrow+\infty$, and the other "larger" (see Figure 5). Now we choose the axis of $\mathbf{M}^{4}$-revolution for the 2-torus to be the 2-plane in Figure 4. It follows that the light-like hypersurface $\mathrm{LL}^{i}\left(T^{2}\right)$ corresponding to the "smaller" Gauss image above has a nonsurjective Gauss map.

\section{APPENDIX 4}

Our purpose here it to show that the intrinsic conformal structure is (generically) a complete local isometry invariant for the induced metric $\overline{\mathrm{I}}=i^{*}\langle\rangle$ of a light-like hypersurface $(N, i)$. We begin with the most degenerate cases. 

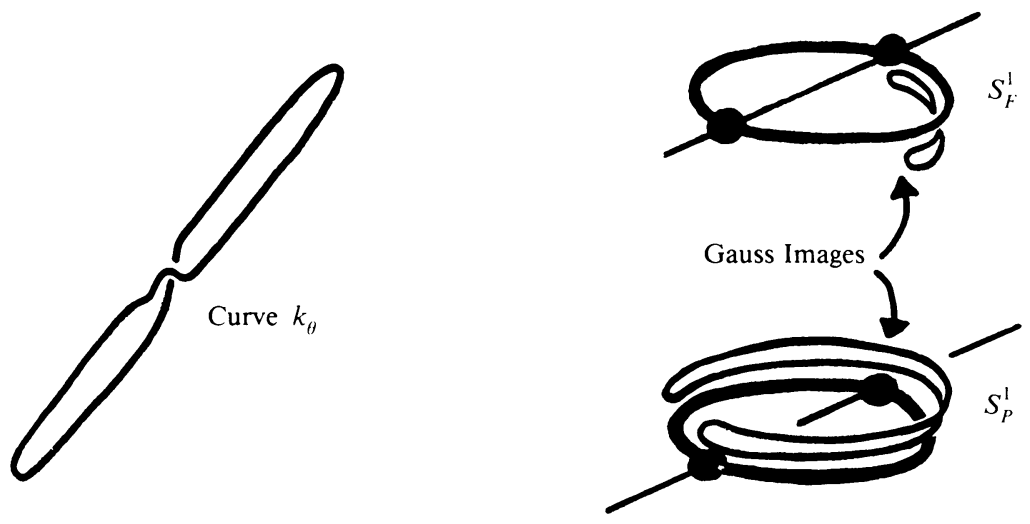

FIGURE 5

Theorem 18. Given $(N, \overline{\mathrm{I}})$ with $\operatorname{Rad}(\overline{\mathbf{I}})$ one dimensional:

(a) if $\overline{\mathrm{II}}$ is a nonzero multiple of $\overline{\mathrm{I}}$ on a neighborhood of $p \in N$, then there exist local coordinates on $N$ such that $\overline{\mathrm{I}}$ is represented by

$$
e^{r} c(x, y)\left(d x^{2}+d y^{2}\right)
$$

(b) if $\overline{\mathrm{II}}=0$ on a neighborhood of $p \in N$ then $\overline{\mathrm{I}}$ is locally represented by

$$
c(x, y)\left(d x^{2}+d y^{2}\right) .
$$

Proof. (a) By assumption there locally exists a vector field $R$ spanning $\operatorname{Rad}(\overline{\mathbf{I}})$ such that $\overline{\mathrm{II}}(-,-, R)=\overline{\mathrm{I}}(-,-)$. Now choose flow adapted coordinates $\left(u_{1}, u_{2}, r\right)$ for $R$ (so that $R$ is represented by $\partial r$ ). In these coordinates $\overline{\mathrm{II}}\left(\partial u_{i}, \partial u_{j}, R\right)=$ $\partial r \overline{\mathrm{I}}\left(\partial u_{i}, \partial u_{j}\right)$. Thus $\overline{\mathrm{I}}$ is represented by $e^{r} g_{i j}\left(u_{1}, u_{2}\right) d u_{i} d u_{j}$ and choosing isothermal coordinates $x, y$ in the slice $r=0$ we are finished.

(b) Follows by a similar argument.

We note that both examples admit a pseudogroup (germ) of isometries. At the other extreme we will now identify conditions on $(N, \overline{\mathrm{I}})$ which enable us to invariantly frame $N$. With a computation similar to those of Proposition 6 we find that the Lie derivative of $\bar{K}$ in the direction of $\bar{R}$ may be written

$$
\bar{R}(\bar{K})=\frac{8 K H\left(H^{2}-K\right)}{\|\mathbf{I I}\|^{3}}(p) .
$$

Now we observe that if $\bar{K}, \bar{H}$ and $\bar{H}^{2}-\bar{K}$ are not zero at $p \in N$ then $d \bar{K} \neq$ 0 at $p$. Hence there exists a unique framing $U_{1}, U_{2}, \bar{R}$ of $N$ near $p$, such that $U_{1}, U_{2}$ are the principal directions of $\overline{\mathrm{II}}(-,-, R)$ with $U_{i}(\bar{K})=0, i=1,2$. Thus a local isometry is completely determined by the condition that it preserve this framing. One might hope that $\bar{K}=$ constant would generate a global foliation of $N$ by sections of $\pi: N \rightarrow M$ (in analogy to the stricture curve of a ruled surface in $\mathbf{E}^{3}$ ). Regrettably Theorem 11 obstructs this possibility. 


\section{BIBLIOGRAPHY}

[B] J. K. Beem and P. E. Ehrlich, Global Lorentzian geometry, Dekker, 1981.

[B] R. Bryant (personal communication).

[K1] M. Kossowski, Fold singularities in pseudo-Riemannian geodesic tubes, Proc. Amer. Math. Soc. 95 (1985), 463-469.

[K2] __ Pseudo-Riemannian metric singularities and the extendability of parallel transport, Proc. Amer. Math. Soc. 99 (1987), 147-154.

[K3] _ The $S^{2}$-valued Gauss maps and split total curvature of a space-like codimension 2 surface in Minkowski space (preprint).

[K4] __ A Gauss map and hybrid degree formula for compact hypersurfaces in Minkowski space (preprint).

[K5] _ Intersection in the space-like Grassmannian and the tilt of a space-like surface (preprint).

[ON] B. O'Neil, Semi Riemannian geometry with applications to relativity, Academic Press, New York, 1983.

[P] R. Penrose, Battile recontates (DeWitt and Wheeler, ed.), Benjamin, New York, 1968.

[P] _ _ General relativity (O'Raifeartaigh, ed.), Clarendon Press, Oxford, 1972.

Department of Mathematics, University of South Carolina, Columbia, South CarOLINA 29208 